\title{
General assembly of FARA in Johannesburg
}

The fourth general assembly of FARA, the Forum for Agricultural Research in Africa, was held from the 10th to 16th of June 2007 in Johannesburg (South Africa).

It was a key event for Africa, where agriculture fortunately is being seen again as a major driver of the economic development of this continent.

It was very interesting to see farmers' associations, NGOs and the private sector fully interacting with researchers during side events and plenary sessions, clearly demonstrating that the concept of Integrated Agricultural Research for Development (IAR4D) is becoming reality.

In the face of certain reductionist views claiming that biotechnologies, often seen as being transferred from North to South, are the only way of saving Africa from hunger and poverty, other views in line with those of IAR4D are emerging. They could lead to rapid, positive changes if based on mutual respect, trust and balanced ownership, and if endorsed by politicians, donors and all stakeholders from producers to consumers, with agriculture being seen as a multifunctional component of sustainable development together with nutrition, health, the environment, biodiversity, urbanization and trade.

As an example, one side event was organized on Africa's agricultural biodiversity, considered to be a hidden wealth for development, nutrition and poverty alle- viation. In this respect, the launching of the Agricultural Biodiversity Initiative for Africa (ABIA) was recognized as a priority for action. Fruits are a major part of this rich biodiversity.

Capturing indigenous knowledge for development, while respecting intellectual property rights, ethical and moral integrity and bio-security, was the central concern of one plenary session. And we know just how rich African knowledge is in terms of the use of fruits for nutrition and traditional medicine.

During the side event on urban horticulture, COorganized by GlobalHort and Urban-Harvest, alarming figures were presented on the very low availability of fruits and vegetables for consumption in sub-Saharan Africa, far below the WHO's critical level of $400 \mathrm{~g}$ per day, particularly in Sahelian countries. A few countries in humid forest zones do reach this level, essentially due to banana and plantain.

Innovation for and in Africa, as seen as a holistic approach for addressing key challenges, must be promoted and enhanced. However, except in a few countries, there is a huge need for capacity building and information exchange, that are the foundations of this vital edifice called "Research for Development".

Dr. Jacky Ganry Chairman of the Section on Tropical and Subtropical Fruits ISHS 


\section{Éditorial}

\section{Assemblée générale du FARA à Johannesburg}

La quatrième assemblée générale du FARA, le forum pour la recherche agricole en Afrique, s'est tenue du 10 au 16 juin 2007 à Johannesburg (Afrique du Sud).

Ce fut un événement essentiel pour l'Afrique où l'agriculture est heureusement considérée à nouveau comme un élément fondamental du développement économique de ce continent.

Ce fut très intéressant de voir les associations paysannes, les ONG (organisations non gouvernementales), le secteur privé interagissant fortement avec les chercheurs durant les sessions parallèles ou en séances plénières, indiquant clairement que le concept de Recherche agricole intégrée pour le développement (IAR4D) devient réalité.

Prenant à contrepied certaines visions réductionnistes considérant les biotechnologies, souvent transférées du Nord au Sud, comme la voie unique pour sauver l'Afrique de la faim et de la pauvreté, d'autres visions, dans la ligne de IAR4D se font jour. Elles pourraient conduire à des évolutions positives rapides à condition d'être basées sur un respect mutuel, sur la confiance et sur une appropriation équilibrée, tout en étant partagées par les politiques, les bailleurs de fonds et l'ensemble des acteurs depuis le producteur jusqu'au consommateur, l'agriculture étant considérée comme une composante multifonctionnelle du développement durable aux cotés de la nutrition, de la santé, de l'environnement, de la biodiversité, de l'urbanisation et du commerce.

A titre d'exemple, une session parallèle a été organisée autour de la biodiversité agricole en Afrique, qui est considérée comme une richesse cachée pour le développement, la nutrition et la lutte contre la pauvreté. A ce titre, le lancement d'une Initiative sur la diversité agricole en Afrique (ABIA) a été retenu comme une priorité pour l'action.

De même, la collecte des savoir-faire locaux en respectant les droits de propriété, l'intégrité éthique et morale, et la biosécurité a fait l'objet d'une attention particulière en session plénière. Et nous savons combien sont riches les savoirs faire africains en termes d'utilisation des fruits pour l'alimentation et la médecine traditionnelle.

Durant la session parallèle sur l'agriculture urbaine, co-organisée par GlobalHort et Urban Harvest, des chiffres alarmants ont été présentés sur la très faible disponibilité en fruits et légumes en Afrique subsaharienne, se situant bien en dessous du seuil critique de $400 \mathrm{~g} /$ jour indiqué par l'OMS, en particulier dans les pays sahéliens. Quelques rares pays d'Afrique forestière humide atteignent ce niveau, en raison de la place importante de la banane et du plantain dans ces pays.

Il apparaît nécessaire de promouvoir et renforcer l'innovation en et pour l'Afrique en l'inscrivant dans une approche intégrée pour faire face aux défis majeurs. Mais, à l'exception de quelques rares pays, il y a un besoin énorme de renforcer les capacités, qui sont les fondations de cet édifice essentiel appelé "Recherche pour le Développement ".

Dr. Jacky Ganry

Président de la section Fruits tropicaux et subtropicaux

ISHS 